\title{
Stem cells, cancer, and cancer stem cells
}

\author{
Tannishtha Reya*§\|, Sean J. Morrison† $\|$, Michael F. Clarkeł \& Irving L. Weissman* \\ *D epartments of Pathology and D evelopmental Biology, Stanford U niversity School of M edicine, Palo Alto, California 94305, USA \\ (e-mail: irv@stanford.edu) \\ †H oward Hughes M edical Institute, and D epartment of Internal M edicine, University of M ichigan, Ann Arbor, M ichigan 48109-0934, USA \\ $¥ D$ epartment of Internal M edicine, University of M ichigan, Ann Arbor, M ichigan 48109-0936, USA \\ $\S$ Present address: D epartment of Pharmacology and Cancer Biology, D ukeUniversity M edical Center, D urham, North Carolina 27710, USA \\ \|Theseauthors contributed equally to this work
}

Stem cell biology has come of age. Unequivocal proof that stem cells exist in the haematopoietic system has given way to the prospective isolation of several tissue-specific stem and progenitor cells, the initial delineation of their properties and expressed genetic programmes, and the beginnings of their utility in regenerative medicine. Perhaps the most important and useful property of stem cells is that of self-renewal. Through this property, striking parallels can be found between stem cells and cancer cells: tumours may often originate from the transformation of normal stem cells, similar signalling pathways may regulate self-renewal in stem cells and cancer cells, and cancer cells may include 'cancer stem cells' - rare cells with indefinite potential for self-renewal that drive tumorigenesis.

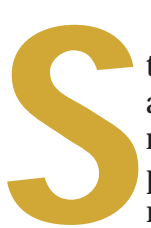

tem cells are defined as cells that have the ability to perpetuate themselves through selfrenewal and to generate mature cells of a particular tissue through differentiation. In most tissues, stem cells are rare. As a result, stem cells must be identified prospectively and purified carefully in order to study their properties. Although it seems reasonable to propose that each tissue arises from a tissue-specific stem cell, the rigorous identification and isolation of these somatic stem cells has been accomplished only in a few instances. For example, haematopoietic stem cells (HSCs) have been isolated from mice and humans $\mathrm{s}^{1-4}$, and have been shown to be responsible for the generation and regeneration of the blood-forming and immune (haematolymphoid) systems (Fig. 1). Stem cells from a variety of organs might have the potential to be used for therapy in the future, but HSCs the vital elements in bone marrow transplantation - have already been used extensively in therapeutic settings (reviewed in ref. 5).

The recent discovery that bone marrow ${ }^{6-8}$, as well as purified $\mathrm{HSC}^{9,10}$, can give rise to non-haematopoietic tissues suggests that thesecells may havegreater differentiation potential than was assumed previously. Definitive experimentsareneeded to determinewhether thecellsfrom the bone marrow that are capable of giving rise to different non-haematopoietic lineages are indeed HSCs or another population. If further studies support the idea of HSC plasticity, this will undoubtedly open new frontiers for understanding the developmental potential of $\mathrm{HSC}$, as well as expand their therapeutic application.

As the characteristics of HSCs, their differentiation potential and clinical applicationshavebeen covered in earlier reviews, here we discuss emerging evidencethat stem cell biology could provide new insights into cancer biology. In particular, we focus on three aspects of the relationship between stem cells and tumour cells: first, the similarities in the mechanisms that regulate self-renewal of normal stem cellsand cancer cells; second, thepossi bility thattumour cells mightarisefrom normal stem cells; and third, thenotion that tumours might contain 'cancer stem cells' - rare cells with indefinite proliferative potential that drive the formation and growth of tumours. Through much of this review we focus on the haematopoietic system because both normal stem cells and cancer cells from this tissueare well characterized. M oreover, cancers of the haematopoietic system (that is, leukaemias) provide the best evidence that normal stem cells are the targets of transforming mutations, and that cancer cell proliferation isdriven by cancer stem cells.

\section{Self-renewal of haematopoietic stem cells}

One of the most important issues in stem cell biology is understanding the mechanisms that regulate self-renewal. Self-renewal is crucial to stem cell function, because it is required by many types of stem cells to persist for the lifetime of the animal. M oreover, whereas stem cells from different organs may vary in their developmental potential, all stem cells must self-renew and regulate the relative balance between self-renewal and differentiation. Understanding the regulation of normal stem cell self-renewal is also fundamental to understanding theregulation of cancer cell proliferation, because cancer can be considered to be a disease of unregulated self-renewal.

In the haematopoietic system, stem cells are heterogeneous with respect to their ability to self-renew. Multipotent progenitors constitute $0.05 \%$ of mouse bone marrow cells, and can be divided into three different populations: longterm self-renewing $\mathrm{HSC}$, short-term self-renewing $\mathrm{HSCS}$, and multipotent progenitors without detectable self-renewal potential ${ }^{2,11}$. These populations form a lineage in which the long-term HSCs giverise to short-term HSCs, which in turn giverise to multipotent progenitors $\mathrm{s}^{11}$. As HSCs maturefrom thelong-term self-renewing pool to multipotent progenitors, they progressively lose their potential to self-renew but become more mitotically active. Whereas long-term HSCs giverise to maturehaematopoietic cellsfor thelifetime of the mouse, short-term HSCs and multipotent progenitors reconstitutelethally irradiated micefor lessthan eightweeks.

Although the phenotypic and functional properties of HSCs have been extensively characterized (reviewed in 
Figure 1 Development of haematopoietic stem cells. HSCs can be subdivided into long-term selfrenewing HSCs, short-term selfrenewing HSCs and multipotent progenitors (red arrows indicate selfrenewal). They give rise to common lymphoid progenitors (CLPs; the precursors of all lymphoid cells) and common myeloid progenitors (CMPs; the precursors of all myeloid cells). Both CMPs/GMPs (granulocyte macrophage precursors) and CLPS can give rise to all known mouse dendritic cells. The isolation of precursors in the haematopoietic system has allowed the generation of a series of mouse models for myeloid leukaemia (see box, lower left). The expression of the oncogenes BCL-2, $B C R-A b l$ and PML- RAR $\alpha$ under the control of the hMRP8 promoter, individually or together, and in combination with Fas deficiency, results in diseases that resemble several human leukaemias, including chronic myelomonocytic leukaemia (CMML), acute myeloid leukaemia (AML), acute promyelocytic leukaemia (APML) $)^{77}$, and chronic myeloid leukaemia (CML)/Blast (S. J aswal, K. Akashi and I.L.W., submitted). ErP, erythrocyte precursor; MEP, megakaryocyte erythrocyte precursor; MkP, megakaryocyte precursor; NK, natural killer.

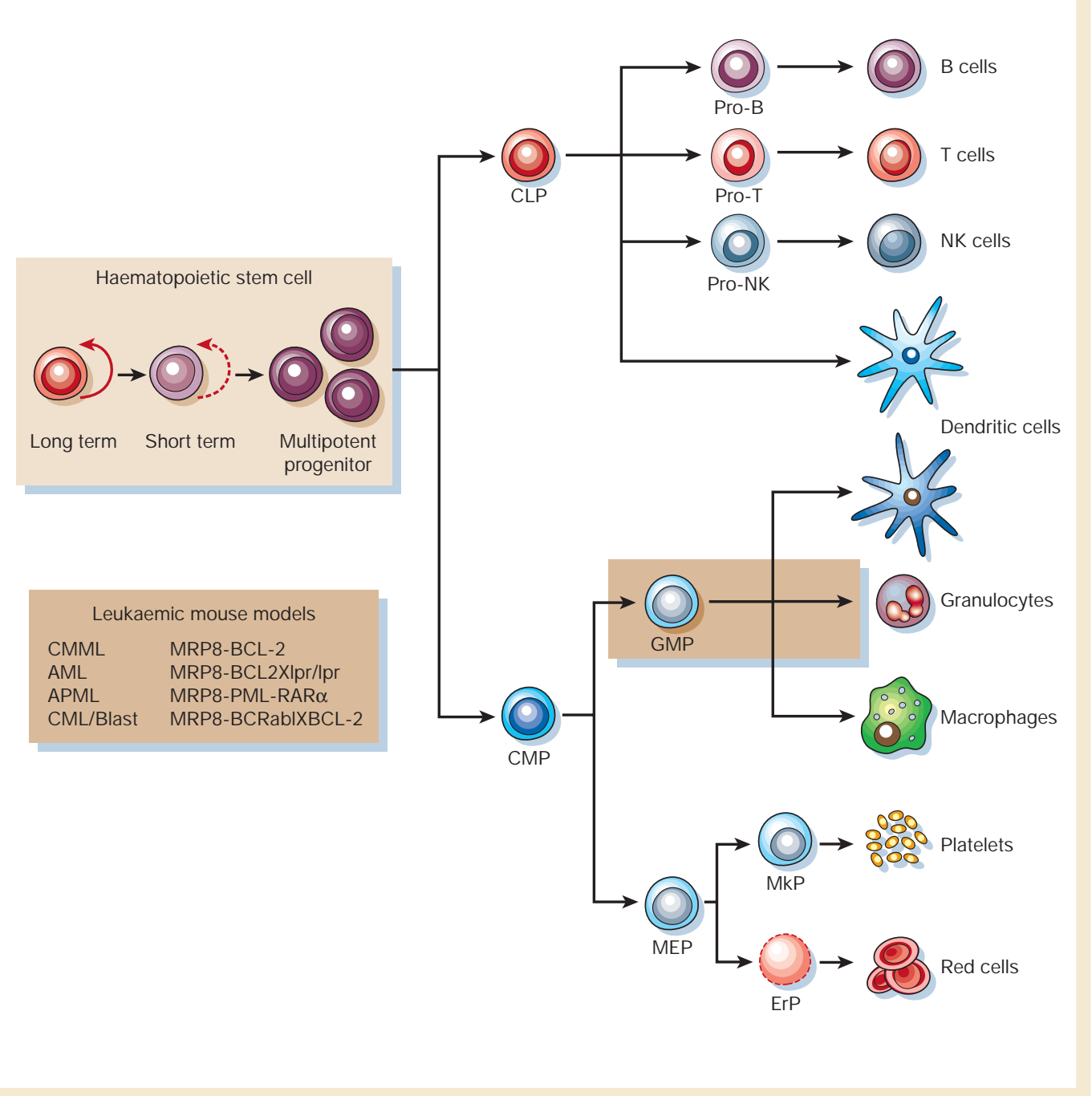

ref. 12), the fundamental question of how self-renewal is regulated remains unanswered. In most cases, combinations of growth factors that can induce potent proliferation cannot prevent the differentiation of HSCs in long-term cultures. Although progress has been madein identifying cultureconditionsthat maintain H SC activity in culture(for example, seeref. 13), it hasproved exceedingly difficult to identify combinations of defined growth factors that cause a significant expansion in culture in the number of progenitors with transplantableH SC activity.

Pathways regulating stem cell self-renewal and oncogenesis Because normal stem cells and cancer cells share the ability to selfrenew, it seems reasonableto propose that newly arising cancer cells appropriatethemachinery for self-renewing cell division that isnormally expressed in stem cells. Evidence shows that many pathways that are classically associated with cancer may also regulate normal stem cell development (Fig. 2). For example, the prevention of apoptosis by enforced expression of the oncogene bcl-2 results in increased numbers of H SC s in vivo, suggesting that cell death has a rolein regulating the homeostasis of $\mathrm{HSC} \mathrm{S}^{14,15}$.

Other signalling pathways associated with oncogenesis, such as the Notch, Sonic hedgehog (Shh) and Wnt signalling pathways, may also regulate stem cell self-renewal (reviewed in ref. 16). N otch activation in H SC s in culture using theligand J agged-1 haveconsistently increased the amount of primitive progenitor activity that can be observed in vitro and in vivo, suggesting that Notch activation promotes HSC self-renewal, or at least the maintenance of multipotentiality ${ }^{17,18}$. Shh signalling has also been implicated in the regulation of self-renewal by the finding that populations highly enriched for human $\mathrm{H} \mathrm{SCS}\left(C D 34^{+} \mathrm{Lin}^{-} \mathrm{CD} 38^{-}\right)$exhibit increased selfrenewal in responseto Shh stimulation in vitro, al beit in combination with other growth factors ${ }^{19}$. Theinvolvement of N otch and Shh in the self-renewal of H SCs is especially interesting in light of studies that implicate these pathways in the regulation of self-renewal of stem cells from other tissues as well (Fig. 2, and see review in this issue by Spradlingand colleagues, pages 98-104).

One particularly interesting pathway that has also been shown to regulate both self-renewal and oncogenesis in different organs is the W nt signalling pathway (Fig. 2). W nt proteins are intercellular signalling molecules ${ }^{20}$ that regulate development in several organisms ${ }^{21}$ and contributeto cancer when dysregulated. Theexpression of W nt proteins in the bone marrow ${ }^{22}$ suggests that they may influence HSCS as well. Using highly purified mouse bone-marrow HSCs, we haveshown that overexpression of activated $\beta$-catenin (a downstream activator of the $\mathrm{W}$ nt signalling pathway) in long-term cultures of HSC s expands the pool of transplantable H SCs determined by both phenotype (Thy $1.1^{10} \mathrm{Lin}^{-/ / 0} \mathrm{Scal}^{+} \mathrm{C}-\mathrm{kit}^{+}$) and function (ability to reconstitute the haematopoietic system in vivo). Moreover, ectopic expression of Axin, an inhibitor of W nt signalling, leadsto inhibition of H SC proliferation, increased death of H SCs in vitro, and reduced reconstitution in vivo (T.R. et al., submitted). In separatestudies, soluble W nt proteins from conditioned supernatants have also been shown to influence the proliferation of haematopoietic progenitors from mousefetal liver and human bonemarrow ${ }^{23,24}$. 


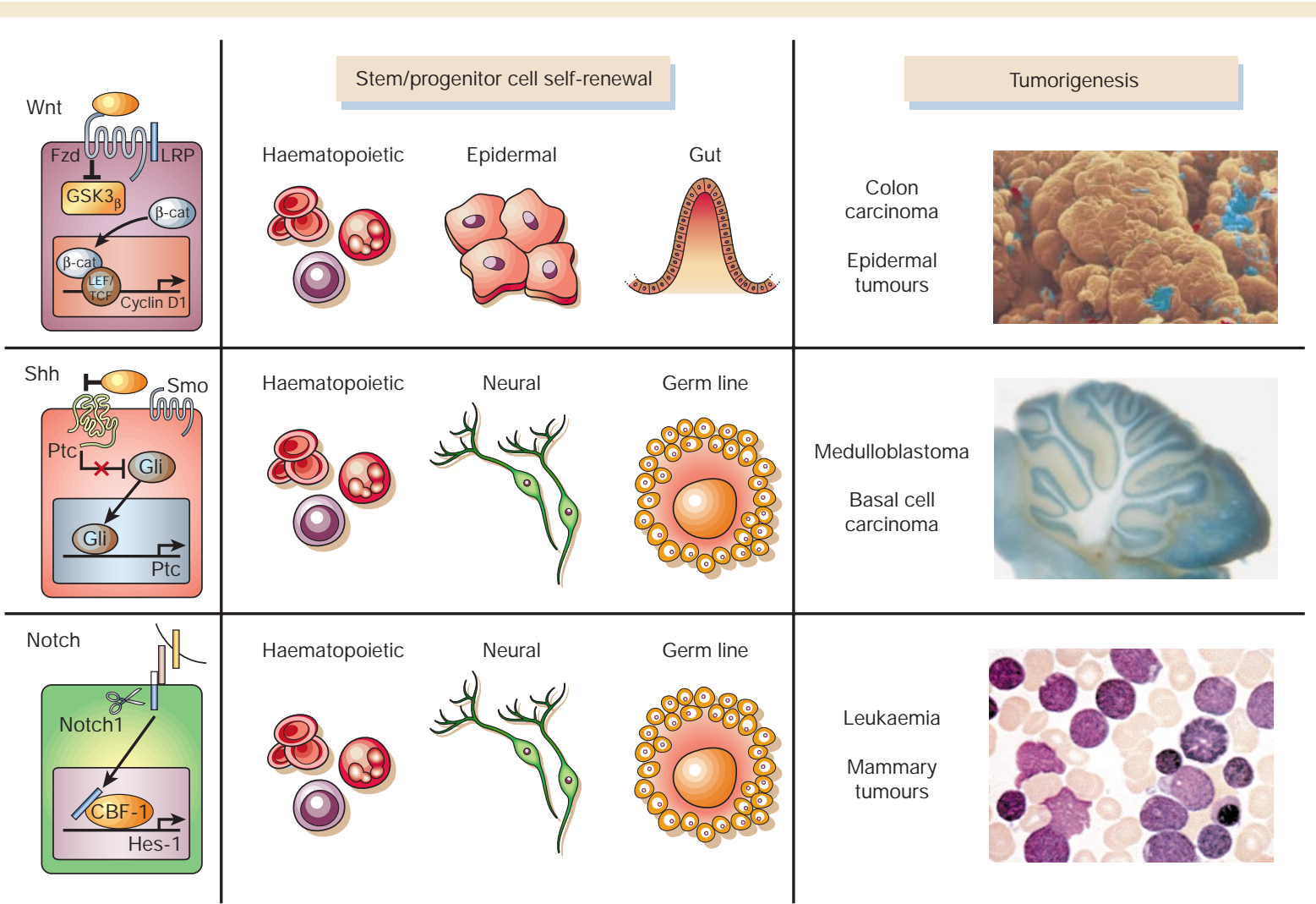

Figure 2 Signalling pathways that regulate self-renewal mechanisms during normal stem cell development and during transformation. Wnt (refs 25, 27; and T.R. et al., submitted), Shh ${ }^{19,78,79}$ and Notch pathways 17,80,81 $^{17}$ have been shown to contribute to the self-renewal of stem cells and/or progenitors in a variety of organs, including the haematopoietic and nervous systems. When dysregulated, these pathways can contribute to oncogenesis. Mutations of these pathways have been associated with a number of human tumours, including colon carcinoma ${ }^{37}$ and epidermal tumours ${ }^{82}$ (Wnt), medulloblastoma ${ }^{83}$ and basal cell carcinoma ${ }^{84}$ (Shh), and T-cell leukaemias ${ }^{85}$ (Notch). (Images courtesy of Eye of Science/SPL and R. Wechsler-Reya/M. Scott/Annual Reviews.)

Studies of epidermal and gut progenitors suggest that the Wnt signalling pathway may contribute to the regulation of stem cell/progenitor cell self-renewal in other tissues. Cultured human keratinocytes with higher proliferativepotential haveincreased levels of $\beta$-catenin compared with keratinocytes with lower proliferative capacity. M oreover, retroviral transduction of activated $\beta$-catenin results in increased epidermal stem cell self-renewal and decreased differentiation ${ }^{25}$. In vivo data from transgenic mice suggest that activation of theW $\mathrm{nt}$ signalling pathwayin epidermal stem cells leads to epithelial cancers $\mathrm{s}^{26}$. Furthermore, mice lacking TCF-4, one of the transcriptional mediators of the Wnt signalling pathway, quickly exhaust the undifferentiated progenitors in the crypts of the gut epithelium during fetal development ${ }^{27}$, suggesting that this pathway is required for the maintenance or self-renewal of gut epithelial stem cells.

Cumulatively, theabove findingssuggest that Wnt signallingmay promote stem cell self-renewal in a variety of different epithelia in addition to HSCs. The molecular mechanisms by which $\mathrm{Wnt}$ signalling influences stem cells remain to be elucidated. It will also be important to determine whether the Wnt, Notch and Shh pathways interact to regulatestem and progenitor cell self-renewal.

\section{Self-renewal and leukaemogenes is}

If the signalling pathways that normally regulate stem cell selfrenewal lead to tumorigenesis when dysregulated, then arestem cells themselvesthetarget of tran sformation in certain types of cancer ${ }^{28,29}$ ? Therearetwo reasons to think that this may bethecase. First, because stem cells have the machinery for self-renewal already activated, maintaining thisactivation may besimpler than turningit on denovo in amoredifferentiated cell; that is, fewer mutationsmay berequired to maintain self-renewal than to activate it ectopically. Second, by self-renewing, stem cells often persist for long periods of time, instead of dying after short periods of timelikemany maturecells in highly proliferative tissues. This means that there is a much greater opportunity for mutations to accumulate in individual stem cells than in most maturecell types (Fig. 3).

Even restricted progenitor cells are less likely than stem cells to undergo neoplastic transformation because they proliferate for a much shorter period of time before terminally differentiating. Restricted haematopoietic progenitors of thelymphoid ${ }^{30}$ and myeloid lineagesall fail to self-renew detectably on tran splantation (K. Nankorn, Traver, D., I.L.W. and K. Akashi, submitted). Thus, restricted progenitorswould firstneed to acquiretheextensiveself-renewal potential of stem cells to have the opportunity to experience additional mutations that would lead to transformation. Nonetheless, restricted progenitors could potentially be transformed either by acquiring mutations that causethem to self-renew like stem cells, or by inheritingexisting mutationsfrom stem cellssuch that onlyasinglemutation is required in theprogenitorsto causetransformation (Fig. 3).

\section{Stem cells as targets of mutation}

For most cancers, the target cell of transforming mutations is unknown; however, thereis considerableevidencethat certain types of leukaemia arise from mutations that accumulate in HSCs. The cells capable of initiating human acutemyeloid leukaemia (AM L) in NOD/SCID (non-obese diabetic/severe combined immunodeficiency) micehavea $C D 34^{+} C D 38^{-}$phenotypein most AM L subtypes, and thus have a phenotype similar to normal $\mathrm{HSCs}^{31}$. Conversely, 
$\mathrm{CD} 34^{+} \mathrm{CD} 38^{+}$leukaemia cells cannot transfer disease to mice in the vast majority of cases, despite the fact that they exhibit a leukaemic blast phenotype. This suggests that normal HSCS rather than committed progenitorsarethetarget for leukaemic transformation.

The most frequent chromosomal abnormalities in AM L involve the 8;21 translocation, which results in AML1-ETO chimaeric transcripts in leukaemic cells. In work done on human H SCs from patients in remission, AML1-ETO transcripts were found in a fraction of normal HSCs in the marrow ${ }^{32}$. These prospectively isolated HSCs and their progeny were not leukaemic, and could differentiate to normal myeloerythroid cells in vitro. This indicates that the translocation occurred originally in normal $\mathrm{HSC}$ s and that additional mutations in a subset of these HSCs or their progeny subsequently lead to leukaemia ${ }^{32}$. In this study, the normal HSCS were $C D 34^{+} C D 38-T h y-1^{+}$, whereas the leukaemic blasts were $\mathrm{CD} 34^{+} \mathrm{CD} 38^{-}$Thy $-1^{-}$. Although the translocation must have occurred in normal $\mathrm{HSC}$, subsequent transforming mutations might haveoccurred either in downstream Thy-1 $1^{-}$progenitors, or in HSC s if one consequence of neoplastic proliferation was the loss of Thy-1 expression. The idea that stem cells are a common target of preleukaemic events or leukaemic transformation isalso supported by work in lymphoid ${ }^{33}$ and chronic myeloid leukaemias ${ }^{34}$ where clonotypic leukaemia-associated chromosomal rearrangements haveal so been found in $C D 34^{+} C D 38^{-}$cells, a population enriched for HSCs. Thus, a variety of leukaemias may arise from mutations that accumulate in HSCs to cause their malignant transformation at the stage of stem cells or their progeny.

\section{Progenitor cells as targets of transformation}

Although stem cells are often the target of genetic events that are necessary or sufficient for malignant transformation, in other cases restricted progenitors or even differentiated cells may becometransformed (Fig. 3). By targetingtheexpression of transgenes specifically to restricted myeloid progenitors using the hM RP- 8 promoter, it is
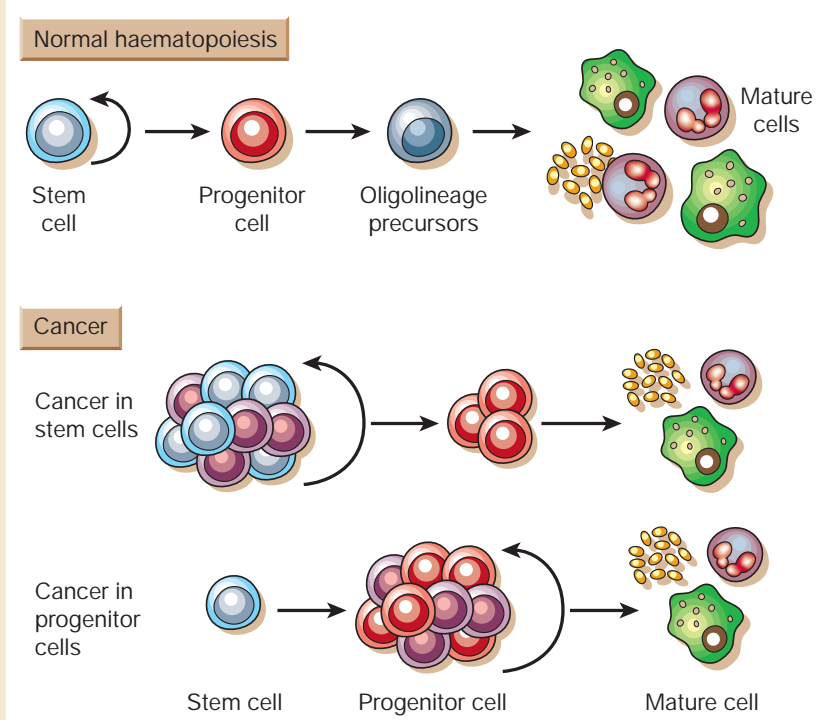

Figure 3 Comparison of self-renewal during haematopoietic stem cell development and leukaemic transformation. Because of their high level of self-renewal, stem cells are particularly good targets of leukaemic transformation. Unlike normal haematopoiesis, where signalling pathways that have been proposed to regulate selfrenewal are tightly regulated (top), during transformation of stem cells, the same mechanisms may be dysregulated to allow uncontrolled self-renewal (middle). Furthermore, if the transformation event occurs in progenitor cells, it must endow the progenitor cell with the self-renewal properties of a stem cell, because these progenitors would otherwise differentiate (bottom). possible to create a mouse model in which myeloid leukaemia arises from restricted progenitors. These leukaemias resemble human leukaemias in many respects, even though the targeted genetic changes cause the leukaemias to arise from restricted progenitors rather than stem cells. For example, we have generated transgenic mouse models for myeloid leukaemias using an hM RP-8 promoter, which targets the expression of transgenes specifically to myeloid progenitor ${ }^{35}$. The enforced expression of the anti-apoptotic gene $\mathrm{bcl}-2$ in themyeloid lineageleads to a diseasethat issimilar to human chronic myelomonocytic leukaemia, including monocytosis, splenomegaly and neutropenia, asthemiceage. However, thesemice rarely develop acutemalignancies.

To test whether additional mutations are required to synergize with bcl-2 to promoteAM L, hM RP 8-bcl-2 transgenic micewerebred with Ipr/lpr Fas-deficient mice. Remarkably, the loss of these two distinct apoptosispathwaysled to the development of AM L in $15 \%$ of the mice ${ }^{36}$. These mice have an expansion of myeloblasts in all haematopoietic tissues, with a substantially lowered number of granulocytes in the marrow and blood. These studies show that prevention of cell death isa crucial event in myeloid leukaemogenesis and that restricted progenitors can be transformed. As described above, in the case of spontaneously arising human leukaemias it is likely that stem cells accumulatethe mutations that are necessary for neoplastic proliferation; however, these mutations may accumulate in stem cells even while the effects of the mutations are expressed in restricted progenitors. That is, mutations that accumulate in stem cells may lead to neoplastic proliferation of primitive progenitors downstream of stem cells.

Perhapsthereason why only $15 \%$ of miceprogress to AM L in mice expressing $\mathrm{BCl}-2$ and lacking Fas is that the progenitors in these mice also must acquire an additional mutation that causes dysregulated self-renewal (Fig. 3). If a singleadditional mutation causes transformation then this transforming event is probably a gain-of-function mutation, such as one that promotes constitutive self-renewal. Because stabilized $\beta$-catenin can promote the self-renewal of HSCs and other types of progenitors (ref. 25, and T.R. et al., submitted; Fig. 2), we propose that gain-of-function mutations in $\beta$-catenin may, in many cases, transform deathless pre-malignant cells to cancer cells by promoting proliferation. In support of thisisevidence to show that activation of $\beta$-catenin and dysregulation of the $\mathrm{Wnt}$ signalling pathway in general is common in cancer ${ }^{37}$, and that the targeted overactivation of this pathway can lead to tumours in transgenicmice ${ }^{38}$. It isal so possiblethat mutationsin other signalling pathways promote progenitor self-renewal. It is important to study this further, because understanding the molecular basis of the unregulated self-renewal of cancer cells will allow the design of more effectivetherapies.

In essence, newly arising cancer cells may appropriate the machinery for self-renewing cell divisions that is normally expressed in stem cells. In the haematopoietic system, the only long-term selfrenewing cells in the myeloerythroid pathway (Fig. 1, bottom) are H SCs; however, at least two differentiated cell types (Fig. 1, top) can also self-renew. Both $T$ and $B$ lymphocytesundergo clonal expansion on stimulation to produce resting memory lymphocytes. These lymphocytes proliferateagain when theantigensarere-encountered. Lymphoid leukaemias can activate these receptor-mediated mitogenic pathways in the course of leukaemogenesi $5^{39-43}$.

\section{Cancer stem cells and aberrant organogenesis}

Basic cancer research has focused on identifying the genetic changes that lead to cancer. This has led to major advances in our understanding of the molecular and biochemical pathways that are involved in tumorigenesis and malignant transformation. But while we have focused on themolecular biology of cancer, our understanding of the cellular biology has lagged. That is, although weunderstand (to a first approximation) the effects of particular mutations on the proliferation and survival of model cells, such as fibroblasts or cell lines, we 
can often only guess what the effects of such mutations will be on the actual cells involved in particular cancers. This has handicapped our ability to translate our identification of mutations into new therapies.

A tumour can be viewed as an aberrant organ initiated by a tumorigenic cancer cell that acquired the capacity for indefinite proliferation through accumulated mutations. If oneviewsa tumour as an abnormal organ, then the principles of normal stem cell biology ${ }^{12,44}$ can beapplied to understand better how tumoursdevelop (reviewed in ref. 45). In fact, many observations suggest that analogies between normal stem cells and tumorigenic cells may be appropriate. Both normal stem cells and tumorigenic cells have extensive proliferative potential and the ability to give rise to new (normal or abnormal) tissues. Both tumours and normal tissues are composed of heterogeneous combinations of cells, with different phenotypic characteristics and different proliferative potentials $s^{46-49}$. Because most tumours have a clonal origi ${ }^{50-52}$, tumorigenic cancer cells must give rise to phenotypically diverse progeny, including cancer cells with indefinite proliferative potential, as well as cancer cells with limited or no proliferative potential. This suggests that tumorigenic cancer cells undergo processes that areanal ogous to the self-renewal and differentiation of normal stem cells.

Although some of the heterogeneity in tumours arises as a result of continuing mutagenesis, it is likely that heterogeneity also arises through the aberrant differentiation of cancer cells. It is well documented that many types of tumours contain cancer cells with heterogeneous phenotypes reflecting aspects of the differentiation that normally occurs in the tissues from which the tumours arise. Thevariableexpression of normal differentiation markers by cancer cellsin a tumour suggests that someof the heterogeneity in tumours arises as a result of the anomal ous differentiation of tumour cells. Examples of this includethevariable expression of myeloid markers in chronic myel oid leukaemia, the variable expression of neuronal markers within peripheral neurectodermal tumours, and the variable expression of milk proteins or the oestrogen receptor within breast cancer.

In other words, both normal stem cellsand tumorigenic cellsgive rise to phenotypically heterogeneous cells that exhibit various degrees of differentiation. Thus, tumorigenic cells can bethought of as cancer stem cells that undergo an aberrant and poorly regulated process of organogenesis anal ogous to what normal stem cellsdo. It is perhapsnot surprising that tumorigenic cells behavein ways that are analogous to normal stem cells given that cancer cellstend to display functional and phenotypic attributes of thenormal cells from which they arederived ${ }^{28}$

\section{Evidence for cancer stem cells}

It was first extensively documented for leukaemia and multiple myelomathat only a small subset of cancer cellsiscapable of extensive proliferation. For example, when mouse myeloma cells were obtained from mouse ascites, separated from normal haematopoietic cells and put in clonal in vitro colony-forming assays, only 1 in 10,000 to 1 in 100 cancer cellswereableto form colonies ${ }^{53}$. Even when leukaemic cells were transplanted in vivo, only $1-4 \%$ of cells could form spleen colonies ${ }^{54-56}$. Because the differences in clonogenicity among theleukaemia cells mirrored the differences in clonogenicity among normal haematopoietic cells, the clonogenic leukaemic cells were described as leukaemic stem cells (for example, seeref. 53). But two formal possibilities remained: either all leukaemia cellshad a low probability of proliferating extensively in these assays such that all leukaemia cellshad thepotential to behaveasleukaemic stem cells, or most leukaemia cellswereunableto proliferateextensively and onlya small, definablesubset of cellswas consistently clonogenic.

To prove the second possibility, it would be necessary to separate different classes of leukaemia cell and show that one subset is highly enriched for clonogenic capacity and all other cells are greatly depleted for clonogenicity. This has been accomplished by Dick and

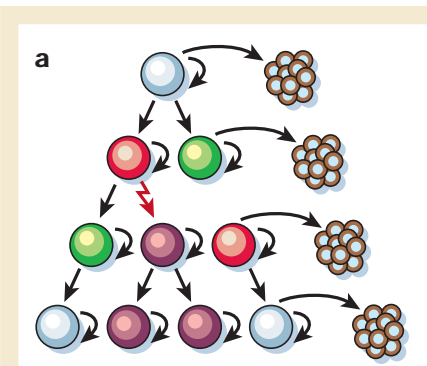
heterogeneous, but most cells can proliferate extensively and form new tumours
Tumour cells are

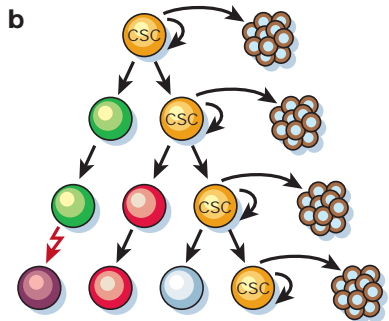

Tumour cells are heterogeneous and only the cancer stem cell subset (CSC; yellow) has the ability to proliferate extensively and form new tumours
Figure 4 Two general models of heterogeneity in solid cancer cells. a, Cancer cells of many different phenotypes have the potential to proliferate extensively, but any one cell would have a low probability of exhibiting this potential in an assay of clonogenicity or tumorigenicity. $\mathbf{b}$, Most cancer cells have only limited proliferative potential, but a subset of cancer cells consistently proliferate extensively in clonogenic assays and can form new tumours on transplantation. The model shown in $\mathbf{b}$ predicts that a distinct subset of cells is enriched for the ability to form new tumours, whereas most cells are depleted of this ability. Existing therapeutic approaches have been based largely on the model shown in a, but the failure of these therapies to cure most solid cancers suggests that the model shown in $\mathbf{b}$ may be more accurate.

colleagues $^{57}$, who showed that human AML stem cells could be identified prospectively and purified as CD $34^{+} C D 38^{-}$cells from patient samples. Despite the fact that these cells represented a small but variableproportion of AM L cells ( $0.2 \%$ in onepatient), they were the only cells capable of transferring $A M L$ from human patients to NOD/SCID micein the vast majority of cases. This excluded the first possibility that all AM L cells had a similar clonogenic capacity, and showed that a small, predictable subset was consistently en riched for theability to proliferateand transfer disease.

It has also been shown for solid cancers that the cells are phenotypically heterogeneous and that only a small proportion of cells are clonogenic in culture and in vivo ${ }^{46-49,58}$. For example, only 1 in 1,000 to 1 in 5,000 lung cancer, ovarian cancer or neuroblastoma cellswere found to form colonies in soft agar ${ }^{59}$. Just as in the context of leukaemic stem cells, these observations led to the hypothesis that only a few cancer cells are actually tumorigenic and that these tumorigenic cells could be considered as cancer stem cells ${ }^{59}$. But, as explained above, two possibilities remain: either all solid cancer cells have a low probability of proliferating extensively and behaving in clonogenicassaysas cancer stem cells, or most cancer cellshaveonlya limited proliferativepotential and cannot behaveas cancer stem cells, but a small, definable subset of cells is enriched for the ability to proliferateextensively and form tumours.

In both cases, some of thecancer cell heterogeneity would ariseas a result of environmental differences within the tumour and continuing mutagenesis. The essential difference between these possibilitiesistheprediction, according to thesecond possibility, that whatever the environment or mutational status of the cells, only a small, phenotypically distinct subset of cancer cells has the ability to prolifer ate extensively or form a new tumour (Fig. 4). It has not been possibleto distinguish between these models of solid cancer heterogeneity, because as yet no one has published theidentity of purified subsets of uncultured solid cancer cells that are enriched for the ability to form new tumours.

The implications of solid cancer stem cells

If the growth of solid cancers were driven by cancer stem cells, it would have profound implications for cancer therapy. At present, all of the phenotypically diverse cancer cells are treated as though they 


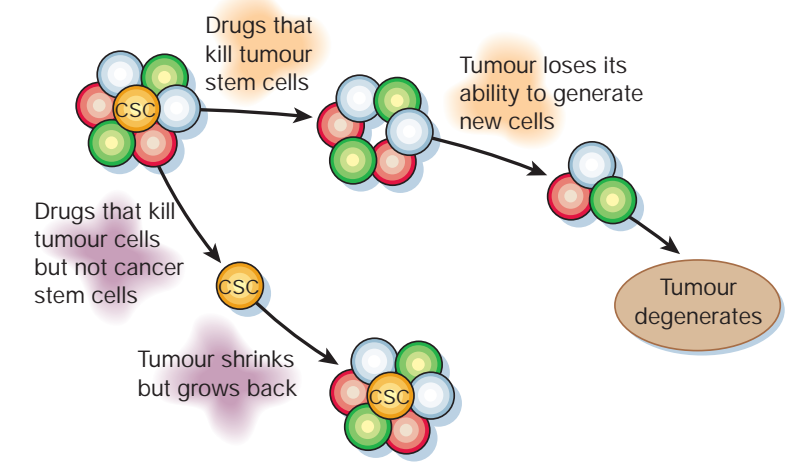

Figure $\mathbf{5}$ Conventional therapies may shrink tumours by killing mainly cells with limited proliferative potential. If the putative cancer stem cells are less sensitive to these therapies, then they will remain viable after therapy and re-establish the tumour. By contrast, if therapies can be targeted against cancer stem cells, then they might more effectively kill the cancer stem cells, rendering the tumours unable to maintain themselves or grow. Thus, even if cancer stem cell-directed therapies do not shrink tumours initially, they may eventually lead to cures.

have unlimited proliferative potential and can acquire the ability to metastasize. For many years, however, it has been recognized that small numbers of disseminated cancer cells can be detected at sites distant from primary tumours in patients that never manifest metastatic disease ${ }^{58,60}$. One possibility is that immune surveillance is highly effective at killing disseminated cancer cells before they can form a detectable tumour. Another possibility is that most cancer cells lack the ability to form a new tumour such that only the dissemination of rare cancer stem cells can lead to metastatic disease ( reviewed in ref. 45). If so, thegoal of therapy must beto identify and kill this cancer stem cell population. If solid cancer stem cells can be identified prospectively and isolated, then we should beableto identify more efficiently new diagnostic markers and therapeutic targets expressed by thestem cells.

If tumour growth and metastasisaredriven by a small population of cancer stem cells, this might explain the failure to develop therapies that are consistently ableto eradicatesolid tumours ${ }^{61}$. Although currently availabledrugscan shrink metastatictumours, theseeffects are usually transient and often do not appreciably extend the life of patients $s^{62,63}$. One reason for the failure of these treatments is the acquisition of drug resistance by the cancer cells as they evolve; another possibility is that existing therapies fail to kill cancer stem cells effectively.

Existing therapies have been developed largely against the bulk population of tumour cells because they are often identified by their ability to shrink tumours. Because most cells with a cancer have limited proliferative potential, an ability to shrink a tumour mainly reflects an ability to kill these cells. It seems that normal stem cells from various tissues tend to be more resistant to chemotherapeutics than maturecell typesfrom thesametissues ${ }^{64}$. Thereasonsfor thisare not clear, but may relateto high levels of expression of anti-apoptotic proteins $^{65-68}$ or $A B C$ transporters such as the multidrug resistance gene ${ }^{69,70}$. If the same were true of cancer stem cells, then one would predict that thesecellswould bemoreresistant to chemotherapeutics than tumour cellswith limited proliferativepotential. Even therapies that cause complete regression of tumours might spare enough cancer stem cells to allow regrowth of the tumours. Therapies that are more specifically directed against cancer stem cells might result in much more durable responses and even cures of metastatic tumours(Fig. 5).

Genomics may provide a powerful means for identifying drug targetsin cancer cells. Although targeting genetic mutations does not requireisolation of thestem cells, therearelikely to bedifferences in gene expression between cancer stem cells and tumour cells with limited proliferative potential. The application of microarray analysis to malignant tumours has shown that patterns of gene expression can be used to group tumours into different categories, often reflecting different mutations ${ }^{71-74}$. As a result, tumour types that cannot be distinguished pathologically, but that can be distinguished on the basis of differences in gene-expression profile, can be examined for differences in treatment sensitivity. However, gene-expression profiling is often conducted on tumour samples that contain a mixture of normal cells, highly proliferative cancer cells, and cancer cells with limited proliferation potential. This results in a composite profile that may obscure differences between tumours, because the highly proliferative cells that drive tumorigenesis often represent a minority of cancer cells. Gene-expression profiling of cancer stem cells would allow the profile to reflect the biology of the cells that are actually driving tumorigenesis. Microdissection of morphologically homogeneous collections of cancer cellsisoneway of generating profilesthat reflect more homogeneous collections of cells $s^{75,76}$. The next frontier will be to purify the cancer stem cells from the whole tumour that retain unlimited proliferative potential and to perform geneexpression profiling on those cells. In addition to being a more efficient way of identifying new therapeutic and diagnostic targets, the profiling of cancer stem cellsmight sharpen the differences in patterns observed between different tumours.

\section{Perspectives}

The ideas discussed in this review can be summarized as a set of propositions. First, self-renewal isthehallmark property of stem cells in normal and neoplastic tissues. Second, in the haematopoietic system, long-term self-renewal islimited to rarelong-term H SC sand some lymphocytes; other cell types lack this potential. Third, cells that continueto divideover long periods of timearemuch more likely to accumulate mutations that cause neoplasia. Thus genetic changes that lead to myeloid leukaemiasmust occur either in long-term HSCs or in progeny that first acquire the ability to self-renew. The fact that normal long-term HSCs in leukaemia patients often have leukaemia-associated translocations strongly supports the idea that leukaemic mutations often accumulate in HSCS. Mutations that lead to certain types of lymphoma may accumulate in lymphocytes, given their ability to self-renew over the longterm. Fourth, in other normal tissuesthat contain self-renewing stem cells, such as the epithelia, the genetic changes that are steps in the progression to solid tumours probably also occur in the stem cells, or in progeny that acquire the potential for self-renewal. Fifth, distinct signalling pathways control stem cell self-renewal in different tissues. But perhaps within individual tissues, the same pathways are used consistently by both normal stem cells and cancer cells to regulate proliferation. For example, W nt signalling regulates the self-renewal of normal stem cells in the blood and epithelia. Constitutive activation of the W nt pathway has been implicated in a number of epithelial cancers. The regulation and consequences of Wnt signalling in normal and neoplastic cells need to be further elucidated. Sixth, understanding thesignal ling pathways that are used by for normal stem cells and neoplastic cells should facilitate the use of normal stem cells for regenerative medicine and theidentification of cancer stem cell targetsfor anticancer therapies. Seventh, within most tumours there may exist cancer stem cells that can self-renew indefinitely, in contrast to most stem cells that may have limited proliferative potential. Finally, in order to cure cancer, it is necessary and sufficient to kill cancer stem cells. To accomplish this it will be necessary to identify and characterize the properties of thesecells.

There are many connections between stem cells and cancer that are important to understand. Just as the signals that are known to control oncogenesis are providing clues about the control of 
self-renewal of normal stem cells, studies of stem cell biology are lending insight into the origins of cancer and will ultimately yield new approaches to fight this disease.

1. Spangrude, G. J., Heimfeld, S. \& Weissman, I. L. Purification and characterization of mouse hematopoietic stem cells. Science 241, 58-62 (1988).

2. Morrison, S. J. \& Weissman, I. L. Thelong-term repopulating subset of hematopoietic stem cells is deterministic and isolatable by phenotype. Immunity 1, 661-673 (1994).

3. Baum, C. M., Weissman, I. L., Tsukamoto, A. S., Buckle, A. M . \& Peault, B. I solation of a candidate human hematopoietic stem-cell population. Proc. Natl Acad. Sci. USA 89, 2804-2808 (1992).

4. Osawa, M., Hanada, K., Hamada, H.\& Nakauchi, H. Long-term lymphohematopoietic reconstitution by a singleCD34-low/negative hematopoietic stem cell. Science 273, 242-245 (1996).

5. Akashi, K. \& Weissman, I. L. in Developmental Biology of Hematopoiesis (ed. Zon, L. I.) 15-34 (Oxford Univ. Press, New York, 2001)

6. Petersen, B. E. et al. Bonemarrow as a potential source of hepatic oval cells. Science 284, 1168-1170 (1999).

7. Brazelton, T. R., Rossi, F. M . V., Keshet, G. I \& Blau, H . M . From marrow to brain: expression of neuronal phenotypes in adult mice. Science 290, 1775-1779 (2000).

8. Mezey, E., Chandross, K. J., H arta, G., M aki, R. A. \& M cKercher, S. R. Turning blood into brain: cells bearing neuronal antigens generated in vivo from bonemarrow. Science 290, 1779-1782 (2000).

9. Lagasse, E. et al. Purified hematopoietic stem cells can differentiate to hepatocytes in vivo. Nature Med. 6, 1229-1234 (2000)

10. Krause, D. S. et al. Multi-organ, multi-lineage engraftment by a single bone marrow derived stem cell. Cell 105, 369-377 (2001).

11. Morrison, S. J., Wandycz, A. M ., Hemmati, H . D., Wright, D. E. \& Weissman, I. L. Identification of a lineage of multipotent hematopoietic progenitors. D evelopment 124, 1929-1939 (1997).

12. Weissman, I. L. Translating stem and progenitor cell biology to the clinic: barriers and opportunities. Science 287, 1442-1446 (2000)

13. Miller, C. L. \& Eaves, C. J. Expansion in vitro of adult murinehematopoietic stem cellswith transplantable lympho-myeloid reconstituting ability. Proc. Natl Acad. Sci. USA 94, 13648-13653 (1997)

14. Domen, J., Gandy, K. L. \& Weissman, I. L. Systemic overexpression of BCL-2 in thehematopoietic system protectstransgenic micefrom the consequences of lethal irradiation. Blood 91, 2272-2282 (1998).

15. Domen, J. \& Weissman, I. L. Hematopoietic stem cells need two signals to prevent apoptosis; BCL-2 can provide one of these, Kitl/c-Kit signaling the other. J. Exp. M ed. 192, 1707-1718 (2000).

16. Tai pale, J. \& Beachy, P. A. The H edgehog and Wnt signaling pathways in cancer. Nature 411 349-354 (2001)

17. Varnum-Finney, B. et al. Pluripotent, cytokine-dependent, hematopoietic stem cells are immortalized by constitutive Notch1 signaling. NatureM ed. 6, 1278-1281 (2000).

18. Karanu, F. N. et al. The Notch ligand Jagged-1 represents a novel growth factor of human hematopoietic stem cells. J. Exp. M ed. 192, 1365-1372 (2000).

19. Bhardwaj, G. et al. Sonic hedgehog induces the proliferation of primitive human hematopoietic cells via BM P regulation. Nature Immunol. 2, 172-180 (2001).

20. Nusse, R. \& Varmus, H. E. M any tumorsinduced by themousemammary tumor virus contain a provirusintegrated in the same region of the host genome. Cell 31, 99-109 (1982).

21. Cadigan, K. M . \& Nusse, R. W nt signaling: a common themein animal development. Genes D ev. 11 3286-3305 (1997).

22. Reya, T. et al. W nt signaling regulates $B$ lymphocyte proliferation through a LEF-1 dependent mechanism. Immunity 13, 15-24 (2000).

23. Austin, T. W., Solar, G. P., Ziegler, F. C., Liem, L. \& M atthews, W. A rolefor theW nt genefamily in hematopoiesis: expansion of multilineage progenitor cells. Blood 89, 3624-3635 (1997).

24. Van Den Berg, D. J., Sharma, A. K., Bruno, E. \& H offman, R. Role of members of theWnt genefamily in human hematopoiesis. Blood 92, 3189-3202 (1998)

25. Zhu, A. J. \& Watt, F. M . $\beta$-catenin signalling modulates proliferative potential of human epidermal keratinocytes independently of intercellular adhesion. Development 126, 2285-2298 (1999).

26. Gat, U., DasGupta, R., Degenstein, L. \& Fuchs, E. Denovo hair folliclemorphogenesis and hair tumors in mice expressing a truncated $\beta$-catenin in skin. Cell 95, 605-614 (1998)

27. Korinek, V. et al. Depletion of epithelia stem-cell compartments in the small intestine of micelacking Tcf-4. Nature Genet. 19, 1-5 (1998).

28. Sell, S. \& Pierce, G. B. M aturation arrest of stem cell differentiation is a common pathway for the cellular origin of teratocarcinomas and epithelial cancers. Lab. Invest. 70, 6-22 (1994).

29. Sawyers, C., Denny, C. \& Witte, O. Leukemia and the disruption of normal hematopoiesis. Cell 64, 337-350 (1991)

30. Kondo, M ., Weissman, I. L. \& Akashi, K. Identification of clonogenic common lymphoid progenitors in mouse bonemarrow. Cell 91, 661-672 (1997).

31. Bonnet, D. \& Dick, J. E. Human acutemyeloid leukemia is organized as a hierarchy that originates from a primitive hematopoietic cell. NatureM ed. 3, 730-737 (1997).

32. M iyamoto, T., Weissman, I. L. \& Akashi, K. AM L1/ETO-expressing nonleukemic stem cells in acute myelogenous leukemia with 8;21 chromosomal translocation. Proc. Natl Acad. Sci. USA 97, 7521-7526 (2000).

33. George, A. A. et al. Detection of leukemic cells in the CD $34^{+} C D 38^{-}$bonemarrow progenitor population in children with acutelymphoblastic leukemia. Blood 97, 3925-3930 (2001).

34. Mauro, M. J. \& Druker, B. J. Chronic myelogenous leukemia. Curr. O pin. Oncol. 13, 3-7 (2001).

35. Lagasse, E. \& Weissman, I. L. bcl-2 inhibits apoptosis of neutrophils but not their engulfment by macrophages. J. Exp. M ed. 179, 1047-1052 (1994)

36. Traver, D., Akashi, K., Weissman, I. L. \& Lagasse, E. Mice defective in two apoptosis pathways in the myeloid lineage develop acutemyeloblastic leukemia. Immunity 9, 47-57 (1998)

37. Polakis, P. W nt signaling and cancer. Genes D ev. 14, 1837-1851 (2000)

38. Tsukamoto, A., Grosschedl, R., Guzman, R., Parslow, T. \& Varmus, H. E. Expression of theint-1 gene in transgenic mice is associated with mammary gland hyperplasia and adenocarcinomas in maleand femalemice. Cell 55, 619-625 (1988).

39. Weissman, I. L. \& Baird, S. in Neoplastic Transformation: M echanisms and Consequences (ed. Korprowski, H.) 135-152 (Dahlem Conferenzen, Berlin, 1977).

40. M CGrath, M. S. \& Weissman, I. L. AKR leukemogenesis: identification and biological significance of thymic lymphoma receptors for AKR retroviruses. Cell 17, 65-75 (1979).

41. M cGrath, M . S., Pillemer, E. \& Weissman, I. L. M urineleukaemogenesis: monoclonal antibodies to Tcell determinants arrest T-lymphoma cell proliferation. Nature 285, 259-261 (1980).
42. Quinn, E. R. et al. The B cell receptor of a hepatitis C virus associated non-H odgkin's lymphoma binds the viral $\mathrm{E}-2$ envelope protein, implicating immunoglobulin activation in lymphomagenesis. Blood (in thepress).

43. O'N eill, H. C., M cGrath, M . S., Allison, J. P. \& Weissman, I. L. A subset of T cell receptors associated with L3T 4 molecules mediates C6V L leukemia cell binding of its cognateretrovirus. Cell 49, 143-151 (1987)

44. M orrison, S. .., Shah, N . M . \& Anderson, D. J. Regulatory mechanisms in stem cell biology. Cell 88, 287-298 (1997).

45. Kummermehr, J. \& Trott, K.-R. in Stem Cells (ed. Potten, C. S.) 363-399 (Academic, New York, 1997)

46. Fidler, I. J. \& Kripke, M. L. M etastasis results from preexisting variant cells within a malignant tumor. Science 197, 893-895 (1977)

47. Fidler, I. J. \& Hart, I. R. Biological diversity in metastatic neoplasms: origins and implications. Science 217, 998-1003 (1982)

48. Heppner, G. H. Tumor heterogeneity. Cancer Res. 44, 2259-2265 (1984).

49. Nowell, P. C. M echanisms of tumor progression. Cancer Res. 46, 2203-2207 (1986)

50. Nowell, P. C. A minute chromosome in human granulocytic leukemia. Science 132, 1497 (1960).

51. Fialkow, P. J. Clonal origin of human tumors. Biochim. Biophys. Acta 458, 283-321 (1976).

52. Fearon, E. R., Hamilton, S. R. \& Vogelstein, B. Clonal analysis of human colorectal tumors. Science 238, 193-197 (1987).

53. Park, C. H., Bergsagel, D. E. \& M cCulloch, E. A. M ousemyeloma tumor stem cells: a primary cell culture assay. J. Natl Cancer Inst. 46, 411-422 (1971).

54. Bruce, W. R. \& Gaag, H. v. d. A quantitativeassay for thenumber of murinelymphoma cells capable of proliferation in vivo. Nature 199, 79-80 (1963).

55. Wodinsky, I., Swiniarski, J. \& Kensler, C. J. Spleen colony studies of leukemia L1210. I. Growth kinetics of lymphocytic L1210 cells in vivo as determined by spleen colony assay. Cancer Chemother. Rep. 51, 415-421 (1967).

56. Bergsagel, D. E. \& Valeriote, F. A. Growth characteristics of a mouse plasma cell tumor. Cancer Res. 28, 2187-2196 (1968).

57. Bonnet, D. \& Dick, J. E. Human acutemyeloid leukemia is organized as a hierarchy that originates from a primitive hematopoietic cell. NatureM ed. 3, 730-737 (1997).

58. Southam, C. M . \& Brunschwig, A. Quantitativestudies of autotransplantation of human cancer. Cancer 14, 971-978 (1961).

59. H amburger, A. W. \& Salmon, S. E. Primary bioassay of human tumor stem cells. Science 197, 461-463 (1977).

60. Salsbury, A. J. The significance of the circulating cancer cell. Cancer Treatment Rev. 2, 55- 72 (1975).

61. Williams, S. D. Treatment of disseminated germ cell tumors with cisplatin, bleomycin, and either vinblastineor etoposide. N. Engl. J. M ed. 316, 1435-1439 (1987).

62. Stockler, M., Wilcken, N. R. C., Ghersi, D. \& Simes, R. J. Systematic reviews of chemotherapy and endocrinetherapy in metastatic breast cancer. Cancer Treatment Rev. 26, 151-168 (2000).

63. Lippman, M. E. High-dosechemotherapy plus autologous bonemarrow transplantation for metastatic breast cancer. N. Engl. J. M ed. 342, 1119-1120 (2000).

64. Harrison, D. E. \& Lerner, C. P. M ost primitive hematopoietic stem cells arestimulated to cycle rapidly after treatment with 5-fluorouracil. Blood 78, 1237-1240 (1991).

65. Bouwens, L. \& DeBlay, E. Islet morphogenesis and stem cell markers in rat pancreas. J. Histochem. Cytochem. 44, 947-951 (1996)

66. Peters, R., Leyvraz, S. \& Perey, L. A poptotic regulation in primitive hematopoietic precursors. Blood 92, 2041-2052 (1998)

67. Domen, J., Gandy, K. L. \& Weissman, I. L. Systemic overexpression of BCL-2 in the hematopoietic system protects transgenic micefrom the consequences of lethal irradiation. Blood 91, 2272-2282 (1998).

68. Feuerhake, F., Sigg, W., Hofter, E. A., Dimpfl, T. \& Welsch, U. Immunohistochemical analysis of Bcl-2 and Bax expression in relation to cell turnover and epithelial differentiation markersin thenonlactating human mammary gland epithelium. Cell TissueRes. 299, 47-58 (2000).

69. Zhou, S. et al. The ABC transporter Bcrp1/ABCG2 is expressed in a wide variety of stem cells and is a molecular determinant of the side-population phenotype. NatureM ed. 7, 1028-1034 (2001).

70. Terskikh, A. V. et al. From hematopoiesis to neuropoiesis: evidence of overlapping genetic programs. Proc. Natl Acad. Sci. USA 98, 7934-7939. (2001).

71. Bittner, M . et al. M olecular classification of cutaneous malignant melanoma by geneexpression profiling. Nature 406, 536-540 (2000)

72. Perou, C. M . et al. M olecular portraits of human breast tumours. Nature 406, 747-752 (2000).

73. Alizadeh, A. A. et al. Distinct types of diffuse large B-cell lymphoma identified by geneexpression profiling. Nature 403, 503-511 (2000).

74. Golub, T. R. Genome-wide views of cancer. N. Engl. J. M ed. 344, 601-602 (2001)

75. Sgroi, D. C. et al. In vivo geneexpression profileanalysis of human breast cancer progression. Cancer Res. 59, 5656-5661 (1999).

76. Leethanakul, C. et al. Distinct pattern of expression of differentiation and growth-related genes in squamous cell carcinomas of the head and neck revealed by the use of laser capturemicrodissection and CDNA arrays. Oncogene 19, 3220-3224 (2000).

77. Kogan, S. C. et al. BCL-2 cooperates with promyelocytic leukemia retinoic acid receptor $\alpha$ chimeric protein (PM LRAR $\alpha$ ) to block neutrophil differentiation and initiate acuteleukemia. J. Exp. M ed. 193, 531-543(2001).

78. Wechsler-Reya, R. J. \& Scott, M . P. Control of neuronal precursor proliferation in the cerebellum by Sonic Hedgehog. Neuron 22, 103-114 (1999).

79. Zhang, Y. \& Kalderon, D. Hedgehog acts as a somatic stem cell factor in the D rosophila ovary. Nature 410, 599-604 (2001).

80. Henrique, D. et al. M aintenance of neuroepithelial progenitor cells by Delta-Notch signalling in the embryonic chick retina. Curr. Biol. 7, 661-670 (1997).

81. Austin, J. \& Kimble, J. glp- 1 is required in the germ linefor regulation of the decision between mitosis and meiosis in C. elegans. Cell 51, 589-599 (1987).

82. Chan, E. F., Gat, U., M CN iff, J. M . \& Fuchs, E. A common human skin tumour is caused by activating mutationsin $\beta$-catenin. Nature Genet. 21, 410-413 (1999).

83. Wechsler-Reya, R. \& Scott, M. P. The developmental biology of brain tumors. Annu. Rev. Neurosci. 24, 385-428 (2001).

84. Gailani, M. R. \& Bale, A. E. Acquired and inherited basal cell carcinomas and the patched gene. Adv. Dermatol. 14, 261-283 (1999).

85. Ellisen, L. W. et al. TAN - 1, the human homolog of theD rosophila notch gene, is broken by chromosomal translocationsin T lymphoblastic neoplasms. Cell 66, 649-661 (1991) 